\title{
SOME RESULTS ON SPACE-LIKE SELF-SHRINKERS
}

\author{
HUAQIAO LIU AND Y. L. XIN
}

\begin{abstract}
We study space-like self-shrinkers of dimension $n$ in pseudo-Euclidean space $\mathbb{R}_{m}^{m+n}$ with index $m$. We derive drift Laplacian of the basic geometric quantities and obtain their volume estimates in pseudo-distance function. Finally, we prove rigidity results under minor growth conditions in terms of the mean curvature or the image of Gauss maps .
\end{abstract}

\section{INTRODUCTION}

Let $\mathbb{R}_{m}^{m+n}$ be an $(m+n)$-dimensional pseudo-Euclidean space with the index $m$. The indefinite flat metric on $\mathbb{R}_{m}^{m+n}$ is defined by $d s^{2}=\sum_{i=1}^{n}\left(d x^{i}\right)^{2}-\sum_{\alpha=n+1}^{m+n}\left(d x^{\alpha}\right)^{2}$. In what follows we agree with the following range of indices

$$
\begin{gathered}
A, B, C, \cdots=1, \cdots, m+n ; i, j, k \cdots=1, \cdots, n ; \\
s, t=1, \cdots, m ; \alpha, \beta, \cdots=n+1, \cdots, m+n .
\end{gathered}
$$

Let $F: M \rightarrow \mathbb{R}_{m}^{m+n}$ be a space-like $n$-dimensional submanifold in $\mathbb{R}_{m}^{m+n}$ with the second fundamental form $B$ defined by $B_{X Y} \stackrel{\text { def. }}{=}\left(\bar{\nabla}_{X} Y\right)^{N}$ for $X, Y \in \Gamma(T M)$. We denote $(\cdots)^{T}$ and $(\cdots)^{N}$ for the orthogonal projections into the tangent bundle $T M$ and the normal bundle $N M$, respectively. For $\nu \in \Gamma(N M)$ we define the shape operator $A^{\nu}: T M \rightarrow T M$ by $A^{\nu}(V)=-\left(\bar{\nabla}_{V} \nu\right)^{T}$. Taking the trace of $B$ gives the mean curvature vector $H$ of $M$ in $\mathbb{R}_{m}^{m+n}$ and $H \stackrel{\text { def. }}{=} \operatorname{trace}(B)=B_{e_{i} e_{i}}$, where $\left\{e_{i}\right\}$ is a local orthonormal frame field of $M$. Here and in the sequel we use the summation convention. The mean curvature vector is time-like, and a cross-section of the normal bundle.

We now consider a one-parameter family $F_{t}=F(\cdot, t)$ of immersions $F_{t}: M \rightarrow$ $\mathbb{R}_{m}^{m+n}$ with the corresponding images $M_{t}=F_{t}(M)$ such that

$$
\begin{aligned}
\frac{d}{d t} F(x, t) & =H(x, t), \quad x \in M \\
F(x, 0) & =F(x)
\end{aligned}
$$

are satisfied, where $H(x, t)$ is the mean curvature vector of $M_{t}$ at $F(x, t)$. There are many interesting results on mean curvature flow on space-like hypersurfaces in certain Lorentzian manifolds [10, 11, 12, 13]. For higher codimension we refer to the previous work of the second author [19].

1991 Mathematics Subject Classification. 58E20,53A10.

The authors are supported partially by NSFC. 
A special but important class of solutions to (1.1) are self-similar shrinking solutions, whose profiles, space-like self-shrinkers, satisfy a system of quasi-linear elliptic PDE of the second order

$$
H=-\frac{X^{N}}{2}
$$

Besides the Lagrangian space-like self-shrinkers [2, 14, 9], there is an interesting paper on curves in the Minkowski plane [15]. The present paper is devoted to general situation on space-like self-shrinker.

For a space-like $n$-submanifold $M$ in $\mathbb{R}_{m}^{m+n}$ we have the Gauss map $\gamma: M \rightarrow$ $\mathbf{G}_{n, m}^{m}$. The target manifold is a pseudo-Grassmann manifold, dual space of the Grassmann manifold $\mathbf{G}_{n, m}$. In the next section we will describe its geometric properties, which will be used in the paper.

Choose a Lorentzian frame field $\left\{e_{i}, e_{\alpha}\right\}$ in $\mathbb{R}_{m}^{m+n}$ with space-like $\left\{e_{i}\right\} \in T M$ and time-like $\left\{e_{\alpha}\right\} \in N M$ along the space-like submanifold $F: M \rightarrow \mathbb{R}_{m}^{m+n}$. Define coordinate functions

$$
x^{i}=\left\langle F, e_{i}\right\rangle, y^{\alpha}=-\left\langle F, e_{\alpha}\right\rangle .
$$

We then have

$$
|F|^{2}=X^{2}-Y^{2}
$$

where $X=\sqrt{\sum_{i=1}^{n}\left(x^{i}\right)^{2}}, \quad Y=\sqrt{\sum_{\alpha=n+1}^{m+n}\left(y^{\alpha}\right)^{2}}$. We call $|F|^{2}$ the pseudo-distance function from the origin $0 \in M$.

We always put the origin on $M$ in the paper. We see that $|F|^{2}$ is invariant under the Lorentzian action up to the choice of the origin in $\mathbb{R}_{m}^{m+n}$. Set $z=|F|^{2}$. It has been proved that $z$ is proper provided $M$ is closed with the Euclidean topology (see [4] for $m=1$ and [16] for any codimension $m$ ).

Following Colding and Minicozzi [6] we can also introduce the drift Laplacian,

$$
\mathcal{L}=\Delta-\frac{1}{2}\langle F, \nabla(\cdot)\rangle=e^{\frac{z}{4}} \operatorname{div}\left(e^{-\frac{z}{4}} \nabla(\cdot)\right) .
$$

It can be showed that $\mathcal{L}$ is self-adjoint with respect to the weighted volume element $e^{-\frac{z}{4}} d \mu$, where $d \mu$ is the volume element of $M$ with respect to the induced metric from the ambient space $\mathbb{R}_{m}^{m+n}$. In the present paper we carry out integrations with respect to this measure. We denote $\rho=e^{-\frac{z}{4}}$ and the volume form $d \mu$ might be omitted in the integrations for notational simplicity.

For a space-like submanifold in $\mathbb{R}_{m}^{m+n}$ there are several geometric quantities. The squared norm of the second fundamental form $|B|^{2}$, the squared norm of the mean curvature $|H|^{2}$ and the $w$-function, which is related to the image of the Gauss map. In $\S 3$ we will calculate drift Laplacian $\mathcal{L}$ of those quantities, see Proposition 3.1 .

Corresponding to the weighted measure and drift Laplacian there is so-called the Baker-Emery Ricci tensor. It is noted that in [3] $\operatorname{Ric}_{f} \geq \frac{z}{4}$ with $f=\frac{z}{4}$. Using the comparison technique the weighted volume of the geodesic ball can be estimated from above in terms of the distance function [18]. 
For a space-like $n$-submanifold $M$ in $\mathbb{R}_{m}^{m+n}$ there are 3 kind global conditions: Closed one with Euclidean topology; entire graph; complete with induced Riemannian metric. A complete space-like one has to be entire graph, but the converse claim is not always the case. Closed one with Euclidean topologg is complete under the parallel mean curvature assumption (see [4] for codimension one and [16] for higher codimension).

In our case of closed one with Euclidean topology, the pseudo-distance function $z$ is always proper. It is natural to consider the volume growth in $z$. For the proper self-shrinkers in Euclidean space Ding-Xin [8] gave the volume estimates. It has been generalized in [5] for more general situation. But, the present case does not satisfy the conditions in Theorem 1.1 in [5]. However, the idea in [8] is still applicable for space-like self-shrinkers. In $\S 4$ we will give volume estimates for space-like selfshrinkers, in a similar manner as in [8], see Theorem 4.1.

Finally, using integral method we can obtain rigidity results as follows.

Theorem 1.1. Let $M$ be a space-like self-shrinker of dimension $n$ in $R_{m}^{n+m}$, which is closed with respect to the Euclidean topology. If there is a constant $\alpha<\frac{1}{8}$, such that $|H|^{2} \leq e^{\alpha z}$, then $M$ is an affine $n$-plane.

Theorem 1.2. Let $M$ be a complete space-like self-shrinker of dimension $n$ in $R_{m}^{n+m}$. If there is a constant $\alpha<\frac{1}{2}$, such that $\ln w \leq e^{\alpha d^{2}(p, x)}$ for certain $p \in M$, where $d(p, \cdot)$ is the distance function from $p$, then $M$ is affine $n-$ plane.

Remark 1.1. In the special situation, for the Lagrangian space-like self-shrinkers, the rigidity results hold without the growth condition (see [9]). Let $\mathbb{R}_{n}^{2 n}$ be Euclidean space with null coordinates $(x, y)=\left(x_{1}, \cdots, x_{n} ; y_{1}, \cdots, y_{n}\right)$, which means that the indefinite metric is defined by $d s^{2}=\sum_{i} d x_{i} d y_{i}$. If $M=\left\{(x, D u(x)) \mid x \in \mathbb{R}^{n}\right\}$ is a space-like submanifold in $\mathbb{R}_{n}^{2 n}$, then $u$ is convex and the induced metric on $M$ is given by $d s^{2}=\sum_{i, j} u_{i j} d x_{i} d x_{j}$. $M$ is a space-like Lagrangian submanifold in $\mathbb{R}_{n}^{2 n}$. It is worthy to point out that if $M$ is entire gradient graph the potential function $u$ is proper, as the following consideration. On $\mathbb{R}^{n}$ set $\rho=|x|=\sqrt{\sum x_{i}^{2}}$. At any direction $\theta \in S^{n-1}$

$$
u_{i}=u_{\rho} \frac{\partial \rho}{\partial x_{i}}=\frac{x_{i}}{\rho} u_{\rho}
$$

and the pseudo-distance

$$
z=x_{i} u_{i}=\rho u_{\rho}
$$

which is positive when the origin is on $M$, since it is space-like. It implies that $u$ is increasing in $\rho$. Moreover,

$$
z_{\rho}=u_{\rho}+\rho u_{\rho \rho}>0
$$

which means that $z$ is also increasing in $\rho$. Hence,

$$
u(\rho)-u(\epsilon)=\int_{\epsilon}^{\rho} u_{\rho} d \rho=\int_{\epsilon}^{\rho} \frac{z}{\rho} d \rho \geq z(\epsilon) \int_{\epsilon}^{\rho} \frac{1}{\rho} d \rho \geq z(\epsilon) \int_{\epsilon}^{\rho} \frac{1}{\rho} d \rho \rightarrow \infty
$$

as $\rho \rightarrow \infty$.

Remark 1.2. Rigidity problem for space-like extremal submanifolds was raised by E. Calabi [1], and solved by Cheng-Yau [4] for codimension 1. Later, Jost-Xin 
generalized the results to higher codimension [16]. The rigidity problem for spacelike submanifolds with parallel mean curvature was studied in [20] [22] and [16] (see also in Chap. 8 of [21]).

\section{Geometry of $G_{n, m}^{m}$}

In $\mathbb{R}_{m}^{n+m}$ all space-like $n$-subspaces form the pseudo-Grassmannian $G_{n, m}^{m}$. It is a specific Cartan-Hadamard manifold which is the noncompact dual space of the Grassmann manifold $G_{n, m}$.

Let $P$ and $A \in G_{n, m}^{m}$ be two space-like $n$-plane in $R_{m}^{m+n}$. The angles between $P$ and $A$ are defined by the critical values of angel $\theta$ between a nonzero vector $x$ in $\mathrm{P}$ and its orthogonal projection $x^{*}$ in $A$ as $x$ runs through $P$.

Assume that $e_{1}, \cdots, e_{n}$ are orthonormal vectors which span the space-like $P$ and $a_{1}, \cdots, a_{n}$ for space-like $A$. For a nonzero vector in $P$

$$
x=\sum_{i} x_{i} e_{i}
$$

its orthonormal projections in $A$ is

$$
x^{*}=\sum_{i} x_{i}^{*} a_{i}
$$

Thus, for any $y \in A$, we have

$$
\left\langle x-x^{*}, y\right\rangle=0
$$

Set

$$
W_{i j}=\left\langle e_{i}, a_{j}\right\rangle
$$

We then have

$$
x_{j}^{*}=\sum_{i} W_{i j} x_{i}
$$

Since $x$ is a vector in a space-like $n$-plane and its projection $x^{*}$ in $A$ is also a spacelike vector. We then have a Minkowski plane $R_{1}^{2}$ spanned by $x$ and $x^{*}$. Then angle $\theta$ between $x$ and $x^{*}$ is defined by

$$
\cosh \theta=\frac{\left\langle x, x^{*}\right\rangle}{|x|\left|x^{*}\right|}
$$

Let

$$
W=\left(W_{i j}\right)=\left(\begin{array}{ccc}
\left\langle e_{1}, a_{1}\right\rangle & \cdots & \left\langle e_{n}, a_{1}\right\rangle \\
\vdots & \vdots & \vdots \\
\left\langle e_{n}, a_{1}\right\rangle & \cdots & \left\langle e_{n}, a_{n}\right\rangle
\end{array}\right)
$$

Now define the $w$-function as

$$
w=\left\langle e_{1} \wedge \cdots \wedge e_{n}, a_{1} \wedge \cdots \wedge a_{n}\right\rangle=\operatorname{det} W .
$$


$W^{T} W$ is symmetric, its eigenvalues are $\mu_{1}^{2}, \cdots, \mu_{n}^{2}$, then there exist $e_{1}, \cdots, e_{n}$ in $P$, such that

$$
W^{T} W=\left(\begin{array}{ccc}
\mu_{1}^{2} & & 0 \\
& \ddots & \\
0 & & \mu_{n}^{2}
\end{array}\right)
$$

in which $\mu_{i} \geq 1$ and $\mu_{i}=\cosh \theta_{i}$. Then

$$
w=\prod_{i} \cosh \theta_{i}=\prod_{i} \frac{1}{\sqrt{1-\lambda_{i}^{2}}}, \lambda_{i}=\tanh \theta_{i} .
$$

The distance between $P$ and $A$ in the canonical Riemannian metric on $\mathbf{G}_{n, m}^{m}$ is (see [17] for example)

$$
d(P, A)=\sqrt{\sum_{i} \theta_{i}^{2}} .
$$

For the fixed $A \in G_{n, m}^{m}$, which is spanned by $\left\{a_{i}\right\}$, choose time-like $\left\{a_{n+s}\right\}$ such that $\left\{a_{i}, a_{n+s}\right\}$ form an orthonormal Lorentzian bases of $R_{m}^{n+m}$.

Set

$$
\begin{aligned}
e_{i} & =\cosh \theta_{i} a_{i}+\sinh \theta_{i} a_{n+i} \\
e_{n+i} & =\sinh \theta_{i} a_{i}+\cosh \theta_{i} a_{n+i}\left(\text { and } e_{n+\alpha}=a_{n+\alpha} \text { if } m>n\right) .
\end{aligned}
$$

Then $e_{i} \in T_{p} M, e_{n+i} \in N_{p} M$. In this case

$$
\begin{aligned}
w_{i \alpha} & =\left\langle e_{1} \wedge \cdots \wedge e_{i-1} \wedge e_{\alpha} \wedge e_{i+1} \cdots \wedge e_{n}, a_{1} \wedge \cdots \wedge a_{n}\right\rangle \\
& =\cosh \theta_{1} \cosh \theta_{i-1} \sinh \theta_{i} \cosh \theta_{i+1} \cosh \theta_{n}=\lambda_{i} w \delta_{n+i \alpha}
\end{aligned}
$$

which is obtained by replacing $e_{i}$ by $e_{\alpha}$ in $w$. We also have $w_{i \alpha j \beta}$ by replacing $e_{j}$ by $e_{\beta}$ in $w_{i \alpha}$. We obtain

$$
w_{i \alpha j \beta}= \begin{cases}\lambda_{i} \lambda_{j} w & \alpha=n+i, \beta=n+j \\ -\lambda_{i} \lambda_{j} w & \alpha=n+j, \beta=n+i \\ 0 & \text { otherwise. }\end{cases}
$$

\section{Drift Laplacian of SOME GEOMETRIC QUANTITIES}

The second fundamental form $B$ can be viewed as a cross-section of the vector bundle $\operatorname{Hom}\left(\odot^{2} T M, N M\right)$ over $M$. A connection on $\operatorname{Hom}\left(\odot^{2} T M, N M\right)$ can be induced from those of $T M$ and $N M$ naturally. There is a natural fiber metric on $\operatorname{Hom}\left(\odot^{2} T M, N M\right)$ induced from the ambient space and it becomes a Riemannian vector bundle. There is the trace-Laplace operator $\nabla^{2}$ acting on any Riemannian vector bundle.

In [19] we already calculate $\nabla^{2} B$ for general space-like $n$-submanifolds in $\mathbb{R}_{m}^{m+n}$.

Set

$$
B_{i j}=B_{e_{i} e_{j}}=h_{i j}^{\alpha} e_{\alpha}, S_{\alpha \beta}=h_{i j}^{\alpha} h_{i j}^{\beta}
$$


From Proposition 2.1 in [19] we have

$$
\left\langle\nabla^{2} B, B\right\rangle=\left\langle\nabla_{i} \nabla_{j} H, B_{i j}\right\rangle+\left\langle B_{i k}, H\right\rangle\left\langle B_{i l}, B_{k l}\right\rangle-\left|R^{\perp}\right|^{2}-\sum_{\alpha, \beta} S_{\alpha \beta}^{2},
$$

where $R^{\perp}$ denotes the curvature of the normal bundle and

$$
\left|R^{\perp}\right|^{2}=-\left\langle R_{e_{i} e_{j}} \nu_{\alpha}, R_{e_{i} e_{j}} \nu_{\alpha}\right\rangle .
$$

Then from the self-shrinker equation (1.2) we obtain

$$
\begin{aligned}
\nabla_{i} F^{N} & =\left[\bar{\nabla}_{i}\left(F-\left\langle F, e_{j}\right\rangle e_{j}\right)\right]^{N} \\
& =\left[e_{i}-\bar{\nabla}_{i}\left\langle F, e_{j}\right\rangle e_{j}-\left\langle F, e_{j}\right\rangle \bar{\nabla}_{e_{i}} e_{j}\right]^{N} \\
& =-\left\langle F, e_{j}\right\rangle B_{i j},
\end{aligned}
$$

and

$$
\begin{aligned}
\nabla_{i} \nabla_{j} F^{N} & =-\nabla_{i}\left[\left\langle F, e_{k}\right\rangle B_{k j}\right] \\
& =-\delta_{i}^{k} B_{k j}-\left\langle F^{N}, B_{k i}\right\rangle B_{k j}-\left\langle F, e_{k}\right\rangle \nabla_{i} B_{k j} \\
& =-B_{i j}-\left\langle F^{N}, B_{k i}\right\rangle B_{k j}-\left\langle F, e_{k}\right\rangle \nabla_{k} B_{i j} \\
& =-B_{i j}+\left\langle 2 H, B_{k i}\right\rangle B_{k j}-\left\langle F, e_{k}\right\rangle \nabla_{k} B_{i j} .
\end{aligned}
$$

Set $P_{i j}=\left\langle B_{i j}, H\right\rangle$, then

$$
\nabla_{i} \nabla_{j} H=\frac{1}{2} B_{i j}-P_{k i} B_{k j}+\frac{1}{2}\left\langle F, e_{k}\right\rangle \nabla_{k} B_{i j} .
$$

Substituting (3.2) into (3.1) we obtain

$$
\begin{aligned}
\left\langle\nabla^{2} B, B\right\rangle= & \left\langle\frac{1}{2} B_{i j}, B_{i j}\right\rangle-\left\langle H, B_{k i}\right\rangle\left\langle B_{k j}, B_{i j}\right\rangle+\frac{1}{2}\left\langle F, e_{k}\right\rangle\left\langle\nabla_{k} B_{i j}, B_{i j}\right\rangle \\
& +\left\langle B_{i k}, H\right\rangle\left\langle B_{i l}, B_{k l}\right\rangle-\left|R^{\perp}\right|^{2}-\sum_{\alpha, \beta} S_{\alpha \beta}^{2} .
\end{aligned}
$$

This also means that

$$
\left\langle\nabla^{2} B, B\right\rangle=\frac{1}{2}\langle B, B\rangle+\frac{1}{4}\left\langle F^{T}, \nabla\langle B, B\rangle\right\rangle-\left|R^{\perp}\right|^{2}-\sum_{\alpha, \beta} S_{\alpha \beta}^{2} .
$$

Note that $\Delta\langle B, B\rangle=2\left\langle\nabla^{2} B, B\right\rangle+2\langle\nabla B, \nabla B\rangle$, so

$$
\begin{aligned}
\Delta\langle B, B\rangle & =\langle B, B\rangle+\frac{1}{2}\left\langle F^{T}, \nabla\langle B, B\rangle\right\rangle-2\left|R^{\perp}\right|^{2}-2 \sum_{\alpha, \beta} S_{\alpha \beta}^{2} \\
& +2\langle\nabla B, \nabla B\rangle .
\end{aligned}
$$

We denote

$$
\begin{gathered}
|B|^{2}=-\langle B, B\rangle=\sum_{i, j, \alpha} h_{\alpha i j}^{2},|\nabla B|^{2}=-\langle\nabla B, \nabla B\rangle . \\
|H|^{2}=-\langle H, H\rangle,|\nabla H|^{2}=-\langle\nabla H, \nabla H\rangle
\end{gathered}
$$

then

$$
\Delta|B|^{2}=|B|^{2}+\frac{1}{2}\left\langle F^{T}, \nabla|B|^{2}\right\rangle+2\left|R^{\perp}\right|^{2}+2 \sum_{\alpha, \beta} S_{\alpha \beta}^{2}+2|\nabla B|^{2}
$$


From (3.2) we also obtain

$$
\nabla^{2} H=\frac{1}{2} H-P_{k i} B_{k j}+\frac{1}{2}\left\langle F, e_{k}\right\rangle \nabla_{k} H .
$$

Since

$$
\Delta|H|^{2}=-\Delta\langle H, H\rangle=-2\left\langle\nabla^{2} H, H\right\rangle-2\langle\nabla H, \nabla H\rangle,
$$

we obtain

$$
\begin{aligned}
\Delta|H|^{2} & =-2\left\langle\frac{1}{2} H-P_{k i} B_{k i}+\frac{1}{2}\left\langle F, e_{k}\right\rangle \nabla_{k} H, H\right\rangle-2\langle\nabla H, \nabla H\rangle \\
& =|H|^{2}+2|P|^{2}+\frac{1}{2}\left\langle F^{T}, \nabla|H|^{2}\right\rangle+2|\nabla H|^{2},
\end{aligned}
$$

where $|P|^{2}=\sum_{i, j} P_{i j}^{2}$.

In the pseudo-Grassmann manifold $\mathbf{G}_{n, m}^{m}$ there are $w$-functions with respect to a fixed point $A \in \mathbf{G}_{n, m}^{m}$, as shown in $\S 2$. For the space-like $n$-submanifold $M$ in $\mathbb{R}_{m}^{m+n}$ we define the Gauss map $\gamma: M \rightarrow \mathbf{G}_{n, m}^{m}$, which is obtained by parallel translation of $T_{p} M$ for any $p \in M$ to the origin in $\mathbb{R}_{m}^{m+n}$. Then, we have functions $w \circ \gamma$ on $M$, which is still denoted by $w$ for notational simplicity.

For any point $p \in M$ around $p$ there is a local tangent frame field $\left\{e_{i}\right\}$, and which is normal at $p$. We also have a local orthonormal normal frame field $\left\{e_{\alpha}\right\}$, and which is normal at $p$. Define a $w$-function by

$$
w=\left\langle e_{1} \wedge \cdots \wedge e_{n}, a_{1} \wedge \cdots \wedge a_{n}\right\rangle,
$$

where $\left\{a_{i}\right\}$ is a fixed orthonormal vectors which span a fixed space-like $n$-plane $A$. Denote

$$
e_{i \alpha}=e_{1} \wedge \cdots \wedge e_{\alpha} \wedge \cdots \wedge e_{n}
$$

which is got by substituting $e_{\alpha}$ for $e_{i}$ in $e_{1} \wedge \cdots \wedge e_{n}$ and $e_{i \alpha j \beta}$ is obtained by substituting $e_{\beta}$ for $e_{j}$ in $e_{i \alpha}$. Then

$$
\begin{aligned}
\nabla_{e_{j}} w & =\sum_{i=1}^{n}\left\langle e_{1} \wedge \cdots \bar{\nabla}_{e_{j}} e_{i} \wedge \cdots \wedge e_{n}, a_{1} \wedge \cdots \wedge a_{n}\right\rangle \\
& =\sum_{i=1}^{n}\left\langle e_{1} \wedge \cdots \wedge B_{i j} \cdots e_{n}, a_{1} \wedge \cdots \wedge a_{n}\right\rangle \\
& =\sum_{i=1}^{n} h_{i j}^{\alpha}\left\langle e_{1} \cdots \wedge e_{\alpha} \wedge \cdots \wedge e_{n}, a_{1} \wedge \cdots \wedge a_{n}\right\rangle \\
& =\sum_{i=1}^{n} h_{i j}^{\alpha}\left\langle e_{i \alpha}, a_{1} \wedge \cdots \wedge a_{n}\right\rangle .
\end{aligned}
$$

Furthermore,

$$
\begin{aligned}
\nabla_{e_{i}} \nabla_{e_{j}} w & =\left\langle\bar{\nabla}_{e_{i}} \bar{\nabla}_{e_{j}}\left(e_{1} \wedge \cdots \wedge e_{n}\right), a_{1} \wedge \cdots \wedge a_{n}\right\rangle \\
& =\sum_{k \neq l}\left\langle e_{1} \wedge \cdots \wedge \bar{\nabla}_{e_{j}} e_{k} \wedge \cdots \wedge \bar{\nabla}_{e_{i}} e_{l} \wedge \cdots \wedge e_{n}, a_{1} \wedge \cdots \wedge a_{n}\right\rangle
\end{aligned}
$$




$$
\begin{aligned}
& +\sum_{k}\left\langle e_{1} \wedge \cdots \bar{\nabla}_{e_{i}} \bar{\nabla}_{e_{j}} e_{k} \wedge \cdots \wedge e_{n}, a_{1} \wedge \cdots \wedge a_{n}\right\rangle \\
= & \sum_{k \neq l}\left\langle e_{1} \wedge \cdots \wedge B_{j k} \wedge \cdots \wedge B_{i l} \wedge \cdots e_{n}, a_{1} \wedge \cdots \wedge a_{n}\right\rangle \\
& +\sum_{k}\left\langle e_{1} \wedge \cdots \wedge\left(\bar{\nabla}_{i} \bar{\nabla}_{j} e_{k}\right)^{T} \wedge \cdots \wedge e_{n}, a_{1} \wedge \cdots \wedge a_{n}\right\rangle \\
& +\sum_{k}\left\langle e_{1} \wedge \cdots \wedge\left(\bar{\nabla}_{i} \bar{\nabla}_{j} e_{k}\right)^{N} \wedge \cdots \wedge e_{n}, a_{1} \wedge \cdots \wedge a_{n}\right\rangle
\end{aligned}
$$

Note that

$$
\begin{aligned}
(\underline{3.8}) & =\sum_{k \neq l} h_{j k}^{\alpha} h_{i l}^{\beta}\left\langle e_{\alpha k \beta l}, a_{1} \wedge \cdots \wedge a_{n}\right\rangle \\
(3.9) & =\left\langle\bar{\nabla}_{i} \bar{\nabla}_{j} e_{k}, e_{k}\right\rangle w=-\left\langle\bar{\nabla}_{j} e_{k}, \bar{\nabla}_{i} e_{k}\right\rangle w=-\left\langle B_{j k}, B_{i k}\right\rangle w=h_{j k}^{\alpha} h_{i k}^{\alpha} w \\
(3.10) & =-\left\langle\left(\bar{\nabla}_{i} \bar{\nabla}_{j} e_{k}\right)^{N}, e_{\alpha}\right\rangle\left\langle e_{\alpha k}, a_{1} \wedge \cdots \wedge a_{n}\right\rangle \\
& =-\left\langle\left(\bar{\nabla}_{i}\left(B_{j k}+\nabla_{e_{j}} e_{k}\right)\right)^{N}, e_{\alpha}\right\rangle\left\langle e_{\alpha k}, a_{1} \wedge \cdots \wedge a_{n}\right\rangle \\
& =-\left\langle\nabla_{i} B_{j k}, e_{\alpha}\right\rangle\left\langle e_{\alpha k}, a_{1} \wedge \cdots \wedge a_{n}\right\rangle=-\left\langle\nabla_{k} B_{i j}, e_{\alpha}\right\rangle\left\langle e_{\alpha k}, a_{1} \wedge \cdots \wedge a_{n}\right\rangle,
\end{aligned}
$$

where we use the Codazzi equation in the last step. Thus, we obtain

$$
\Delta w=\sum_{i, k \neq l} h_{i k}^{\alpha} h_{i l}^{\beta}\left\langle e_{k \beta l}^{\alpha}, a_{1} \wedge \cdots \wedge a_{n}\right\rangle+|B|^{2} w-\left\langle\nabla_{k} H, e_{\alpha}\right\rangle\left\langle e_{\alpha k}, a_{1} \wedge \cdots \wedge a_{n}\right\rangle,
$$

Since

from (1.2), we obtain

$$
\nabla_{i} F^{N}=-\left\langle F, e_{j}\right\rangle B_{i j}
$$

$$
\begin{aligned}
\nabla_{i} H & =\frac{1}{2}\left\langle F, e_{j}\right\rangle B_{i j} \\
\left\langle\nabla_{i} H, e_{\alpha}\right\rangle & =-\frac{1}{2}\left\langle F, e_{j}\right\rangle h_{i j}^{\alpha},
\end{aligned}
$$

So,

$$
\begin{aligned}
\Delta w & =|B|^{2} w+\sum_{i, k \neq l} h_{i k}^{\alpha} h_{i l}^{\beta}\left\langle e_{\alpha k \beta l}, a_{1} \wedge \cdots \wedge a_{n}\right\rangle+\frac{1}{2}\left\langle F, e_{i}\right\rangle h_{k i}^{\alpha}\left\langle e_{\alpha k}, a_{1} \wedge \cdots \wedge a_{n}\right\rangle \\
& =|B|^{2} w+\sum_{i, k \neq l} h_{i k}^{\alpha} h_{i l}^{\beta}\left\langle e_{\alpha k \beta l}, a_{1} \wedge \cdots \wedge a_{n}\right\rangle+\frac{1}{2}\langle F, \nabla w\rangle,
\end{aligned}
$$

where (3.7) has been used in the last equality.

Proposition 3.1. For a space-like self-shrinker $M$ of dimension $n$ in $\mathbb{R}_{m}^{m+n}$ we have

$$
\begin{gathered}
\mathcal{L}|B|^{2}=|B|^{2}+2\left|R^{\perp}\right|^{2}+2 \sum_{\alpha, \beta} S_{\alpha \beta}^{2}+2|\nabla B|^{2}, \\
\mathcal{L}|H|^{2}=|H|^{2}+2|P|^{2}+2|\nabla H|^{2}, \\
\mathcal{L}(\ln w) \geq \frac{|B|^{2}}{w^{2}} .
\end{gathered}
$$


Proof. From (1.3) 3.5.3.6), we can obtain (3.13) and (3.14) easily.

From (1.3.3.12) we have

$$
\begin{aligned}
\mathcal{L} w & =|B|^{2} w+\sum_{i, k \neq l} h_{i k}^{\alpha} h_{i l}^{\beta}\left\langle e_{\alpha k \beta l}, a_{1} \wedge \cdots \wedge a_{n}\right\rangle=|B|^{2} w+\sum_{i, k \neq l} h_{i k}^{\alpha} h_{i l}^{\beta} w_{\alpha k \beta l} \\
& =|B|^{2} w+\sum_{i, k \neq l} \lambda_{k} \lambda_{l}\left(h_{i k}^{n+k} h_{i l}^{n+l}-h_{i k}^{n+l} h_{i l}^{n+k}\right) w
\end{aligned}
$$

Furthermore, since

$$
\mathcal{L}(\ln w)=\frac{1}{w} \mathcal{L} w-\frac{|\nabla w|^{2}}{w^{2}}
$$

we obtain

$$
\mathcal{L}(\ln w)=|B|^{2}+\sum_{i, k \neq l} \lambda_{k} \lambda_{l}\left(h_{i k}^{n+k} h_{i l}^{n+l}-h_{i k}^{n+l} h_{i l}^{n+k}\right)-\frac{|\nabla w|^{2}}{w^{2}} .
$$

From (3.7), we obtain

$$
\begin{aligned}
|\nabla w|^{2} & =\sum_{j=1}^{n}\left|\nabla_{e_{j}} w\right|^{2}=\sum_{j=1}^{n}\left(\sum_{i=1}^{n} \sum_{\alpha} h_{i j}^{\alpha} w_{i \alpha}\right)^{2} \\
& =\sum_{j=1}^{n}\left(\sum_{i=1}^{n} h_{i j}^{n+i} \lambda_{i} w\right)^{2}=\sum_{i, j, k=1}^{n} \lambda_{i} \lambda_{k} w^{2} h_{i j}^{n+i} h_{k j}^{n+k} .
\end{aligned}
$$

in the case of $m \geq n$ we rewrite (otherwise, we treat the situation similarly)

$$
|B|^{2}=\sum_{j, k, \alpha>n}\left(h_{j k}^{n+\alpha}\right)^{2}+\sum_{i, j}\left(h_{i j}^{n+i}\right)^{2}+\sum_{j} \sum_{k<i}\left(h_{i j}^{n+k}\right)^{2}+\sum_{j} \sum_{i<k}\left(h_{i j}^{n+k}\right)^{2} .
$$


So, we obtain

$$
\begin{aligned}
& \mathcal{L}(\ln w)=|B|^{2}+\sum_{i, j, k \neq i} \lambda_{i} \lambda_{k}\left(h_{i j}^{n+i} h_{j k}^{n+k}-h_{i j}^{n+k} h_{j k}^{n+i}\right)-\sum_{i, j, k=1}^{n} \lambda_{i} \lambda_{k} h_{i j}^{n+i} h_{j k}^{n+k} \\
& =|B|^{2}+\sum_{i, j, k \neq i} \lambda_{i} \lambda_{k} h_{i j}^{n+i} h_{j k}^{n+k}-\sum_{i, j, k \neq i} \lambda_{i} \lambda_{k} h_{i j}^{n+k} h_{j k}^{n+i}-\sum_{i, j, k=1}^{n} \lambda_{i} \lambda_{k} h_{i j}^{n+i} h_{j k}^{n+k} \\
& =\sum_{j, k, \alpha>n}\left(h_{j k}^{n+\alpha}\right)^{2}+\sum_{i, j}\left(h_{i j}^{n+i}\right)^{2}+\sum_{j} \sum_{k<i}\left(h_{i j}^{n+k}\right)^{2}+\sum_{j} \sum_{i<k}\left(h_{i j}^{n+k}\right)^{2} \\
& -\sum_{i, j} \lambda_{i}^{2}\left(h_{i j}^{n+i}\right)^{2}-\sum_{i j, k \neq i} \lambda_{i} \lambda_{k} h_{i j}^{n+k} h_{j k}^{n+i} \\
& =\sum_{j, k, \alpha>n}\left(h_{j k}^{n+\alpha}\right)^{2}+\sum_{i, j}\left(1-\lambda_{i}^{2}\right)\left(h_{i j}^{n+i}\right)^{2}+\sum_{j} \sum_{k<i}\left(h_{i j}^{n+k}\right)^{2}+\sum_{j} \sum_{i<k}\left(h_{i j}^{n+k}\right)^{2} \\
& -2 \sum_{j} \sum_{k<i} \lambda_{k} \lambda_{i} h_{j k}^{n+i} h_{i j}^{n+k} \\
& \geq \sum_{j, k, \alpha>n}\left(h_{j k}^{n+\alpha}\right)^{2}+\sum_{i, j}\left(1-\lambda_{i}^{2}\right)\left(h_{i j}^{n+i}\right)^{2}+\sum_{j} \sum_{k<i}\left(1-\lambda_{i}^{2}\right)\left(h_{i j}^{n+k}\right)^{2} \\
& +\sum_{j} \sum_{i<k}\left(1-\lambda_{i}^{2}\right)\left(h_{i j}^{n+k}\right)^{2} \\
& =\sum_{j, k, \alpha>n}\left(h_{j k}^{n+\alpha}\right)^{2}+\sum_{i, j, k}\left(1-\lambda_{i}^{2}\right)\left(h_{i j}^{n+k}\right)^{2} \\
& \geq \sum_{j, k, \alpha>n}\left(h_{j k}^{n+\alpha}\right)^{2}+\prod_{i}\left(1-\lambda_{i}^{2}\right) \sum_{i, j, k}\left(h_{i j}^{n+k}\right)^{2} .
\end{aligned}
$$

Noting (2.1) the inequality (3.15) has been proved.

Remark 3.1. For a space-like graph $M=(x, f(x))$ with $f: \mathbb{R}^{n} \rightarrow \mathbb{R}^{m}$ its induced metric is $d s^{2}=\left(\delta_{i j}-f_{i}^{\alpha} f_{j}^{\alpha}\right) d x^{i} d x^{j}$. Set $g=\operatorname{det}\left(\delta_{i j}-f_{i}^{\alpha} f_{j}^{\alpha}\right)$ then $w=\frac{1}{\sqrt{g}}$.

Remark 3.2. (3.15) is a generalization of a formula (5.8) for space-like graphical self-shrinkers in [7] to more general situation.

\section{Volume GROWTH}

To draw our results we intend to integrate those differential inequalities obtained in the last section. We need to know the volume growth in the pseudo-distance function $z$ on the space-like submanifolds. In [16] the following property has been proved. 
Proposition 4.1. (Proposition 3.1 in [16]) Let $M$ be a space-like $n$-submanifold in $\mathbb{R}_{m}^{m+n}$. If $M$ is closed with respect to the Euclidean topology, then when $0 \in M$, $z=\langle F, F\rangle$ is a proper function on $M$.

we also need a lemma from [8]:

Lemma 4.1. If $f(r)$ is a monotonic increasing nonnegative function on $[0,+\infty)$ satisfying $f(r) \leq C_{1} r^{n} f\left(\frac{r}{2}\right)$ on $\left[C_{2},+\infty\right)$ for some positive constant $n, C_{1}, C_{2}$, here $C_{2}>1$, then $f(r) \leq C_{3} e^{2 n(\log r)^{2}}$ on $\left[C_{2},+\infty\right)$ for some positive constant $C_{3}$ depending only on $n, C_{1}, C_{2}, f\left(C_{2}\right)$.

Using the similar method as in [8] we obtain the following volume growth estimates.

Theorem 4.1. Let $z=\langle F, F\rangle$ be the pseudo-distance of $R_{m}^{n+m}$, where $F \in R_{m}^{n+m}$ is the position vector with respect to the origin $0 \in M$. Let $M$ be an $n$-dimensional space-like self-shrinker of $R_{m}^{n+m}$. Assume that $M$ is closed with respect to the Euclidean topology, then for any $\alpha>0, \int_{M} e^{-\alpha z} d \mu$ is finite, in particular $M$ has finite weighted volume.

Proof. We have

$$
\begin{gathered}
z_{i} \stackrel{\text { def. }}{=} e_{i}(z)=2\left\langle F, e_{i}\right\rangle, \\
z_{i j} \stackrel{\text { def. }}{=} \operatorname{Hess}(z)\left(e_{i}, e_{j}\right)=2\left(\delta_{i j}-y^{\alpha} h_{i j}^{\alpha}\right), \\
\Delta z=2 n-2 y^{\alpha} H^{\alpha}=2 n+Y^{2},
\end{gathered}
$$

where the self-shrinker equation (1.2) has been used in third equality. For our selfshrinker $M^{n}$ in $\mathbb{R}_{m}^{n+m}$, we define a functional $F_{t}$ on any set $\Omega \subset M$ by

$$
F_{t}(\Omega)=\frac{1}{(4 \pi t)^{n / 2}} \int_{\Omega} e^{-\frac{z}{4 t}} d \mu, \quad \text { for } \quad t>0
$$

Set $B_{r}=\left\{p \in \mathbb{R}_{m}^{m+n}, z(p)<r^{2}\right\}$ and $D_{r}=B_{r} \bigcap M$. We differential $F_{t}\left(D_{r}\right)$ with respect to $t$,

$$
F_{t}^{\prime}\left(D_{r}\right)=(4 \pi)^{-\frac{n}{2}} t^{-\left(\frac{n}{2}+1\right)} \int_{D_{r}}\left(-\frac{n}{2}+\frac{z}{4 t}\right) e^{-\frac{z}{4 t}} d \mu
$$

Noting (4.1)

$$
\begin{aligned}
-e^{\frac{z}{4 t}} \operatorname{div}\left(e^{-\frac{z}{4 t}} \nabla z\right) & =-\Delta z+\frac{1}{4 t} \nabla z \cdot \nabla z \\
& =-2 n-Y^{2}+\frac{X^{2}}{t} \\
& \geq \frac{z}{t}-2 n \quad(\text { when } 0<t \leq 1) .
\end{aligned}
$$

Since

$$
\nabla z=2 F^{T}
$$


and the unit normal vector to $\partial D_{r}$ is $\frac{F^{T}}{X}$, then

$$
\begin{aligned}
F_{t}^{\prime}\left(D_{r}\right) & \leq \pi^{-\frac{n}{2}}(4 t)^{-\left(\frac{n}{2}+1\right)} \int_{D_{r}}-\operatorname{div}\left(e^{-\frac{z}{4 t}} \nabla z\right) d \mu \\
& =\pi^{-\frac{n}{2}}(4 t)^{-\left(\frac{n}{2}+1\right)} \int_{\partial D_{r}}-2 X e^{-\frac{z}{4 t}} \leq 0 .
\end{aligned}
$$

We integrate $F_{t}^{\prime}\left(D_{r}\right)$ over $t$ from $\frac{1}{r}$ to $1, r \geq 1$, and get

$$
\int_{D_{r}} e^{-\frac{z}{4}} d \mu \leq r^{\frac{n}{2}} \int_{D_{r}} e^{-\frac{z r}{4}} d \mu,
$$

Since

and

$$
\int_{D_{r}} e^{-\frac{z}{4}} d \mu \geq e^{-\frac{r^{2}}{4}} \int_{D_{r}} 1 d \mu
$$

$$
\begin{aligned}
\int_{D_{r}} e^{-\frac{z r}{4}} d \mu & =\int_{D_{r} \backslash D_{\frac{r}{2}}} e^{-\frac{z r}{4}} d \mu+\int_{D_{\frac{r}{2}}} e^{-\frac{z r}{4}} d \mu \\
& \leq \quad e^{-\frac{r^{3}}{16}} \int_{D_{r}} 1 d \mu+\int_{D_{\frac{r}{2}}} 1 d \mu .
\end{aligned}
$$

Set $V(r)=\int_{D_{r}} 1 d \mu$. Then,

$$
\left(e^{-\frac{r^{2}}{4}}-e^{-\frac{r^{3}}{16}} r^{\frac{n}{2}}\right) V(r) \leq r^{\frac{n}{2}} V\left(\frac{r}{2}\right) .
$$

Let $g(r)=e^{-\frac{r^{2}}{4}}-e^{-\frac{r^{3}}{16}} r^{\frac{n}{2}} \cdot g(r)>0$ when $r$ sufficiently large (say $r \geq 8 n$ ) Since

$$
\begin{aligned}
g^{\prime}(r) & =-\frac{r}{2} e^{-\frac{r^{2}}{4}}-\frac{n}{2} r^{\frac{n}{2}-1} e^{-\frac{r^{3}}{16}}+\frac{3 r^{2}}{16} r^{\frac{n}{2}} e^{-\frac{r^{3}}{16}} \\
& >\left(-\frac{r}{2}-\frac{n}{2} r^{\frac{n}{2}-1}+\frac{3}{16} r^{\frac{n}{2}+2}\right) e^{-\frac{r^{3}}{16}}>0,
\end{aligned}
$$

$g(r)$ is increasing in $r$ and $g^{-1}(r)$ is decreasing in $r$. Therefore,

$$
g^{-1}(r) \leq \frac{1}{e^{-16 n^{2}}-e^{-32 n^{3}}(8 n)^{\frac{n}{2}}}=C_{1}
$$

We then have

$$
V(r) \leq C_{1} r^{n} V\left(\frac{r}{2}\right) \quad \text { for } r \text { sufficiently large (say, } r \geq 8 n \text { ). }
$$

By Lemma 4.1, we have

$$
V(r) \leq C_{4} e^{2 n(\log r)^{2}} \quad \text { for } \quad r \geq 8 n,
$$

here $C_{4}$ is a constant depending only on $n, V(8 n)$. Hence, for any $\alpha>0$

$$
\begin{aligned}
\int_{M} e^{-\alpha z} d \mu & =\sum_{j=0}^{\infty} \int_{D_{8 n(j+1)} \backslash D_{8 n j}} e^{-\alpha z} d \mu \leq \sum_{j=0}^{\infty} e^{-\alpha(8 n j)^{2}} V(8 n(j+1)) \\
& \leq C_{4} \sum_{j=0}^{\infty} e^{-\alpha(8 n j)^{2}} e^{2 n(\log (8 n)+\log (j+1))^{2}} \leq C_{5}
\end{aligned}
$$


where $C_{5}$ is a constant depending only on $n, V(8 n)$. So we obtain our estimates. Certainly, $M$ has weighted finite volume.

Corollary 4.1. Any space-like self-shrinker $M$ of dimension $n$ in $\mathbb{R}_{m}^{m+n}$ with closed Euclidean topology has finite fundamental group.

From the Gauss equation we have

$$
\operatorname{Ric}\left(e_{i}, e_{i}\right)=\left\langle H, B_{i i}\right\rangle-\sum_{j}\left\langle B_{i j}, B_{i j}\right\rangle,
$$

and

$$
\operatorname{Hess}(f)\left(e_{i}, e_{i}\right)=\frac{1}{4} \operatorname{Hess}(z)\left(e_{i}, e_{i}\right)=\frac{1}{2} \delta_{i j}+\frac{1}{2}\left\langle F, B_{i j}\right\rangle=\frac{1}{2} \delta_{i j}-\left\langle H, B_{i j}\right\rangle,
$$

It follows that

$$
\operatorname{Ric}_{f}\left(e_{i}, e_{i}\right)=\operatorname{Ric}\left(e_{i}, e_{i}\right)+\operatorname{Hess}(f)\left(e_{i}, e_{i}\right) \geq \frac{1}{2}
$$

Set $B_{R}(p) \subset M$, a geodesic ball of radius $R$ and centered at $p \in M$. From Theorem 3.1 in [18] we know that for any $r$ there are constant $A, B$ and $C$ such that

$$
\int_{B_{R}(p)} \rho \leq A+B \int_{r}^{R} e^{-\frac{1}{2} t^{2}+C t} d t
$$

\section{Rigidity RESUlts}

Now, we are in a position to prove rigidity results mentioned in the introduction.

Theorem 5.1. Let $M$ be a space-like self-shrinker of dimension $n$ in $R_{m}^{n+m}$, which is closed with respect to the Euclidean topology. If there is a constant $\alpha<\frac{1}{8}$, such that $|H|^{2} \leq e^{\alpha z}$, then $M$ is an affine $n-$ plane.

Proof. Let $\eta$ be a smooth function with compact support in $M$, then by (3.14) we obtain

$$
\begin{aligned}
\int_{M}\left(\frac{1}{2}|H|^{2}+|P|^{2}+|\nabla H|^{2}\right) \eta^{2} \rho & =\frac{1}{2} \int_{M}\left(\mathcal{L}|H|^{2}\right) \eta^{2} \rho=\frac{1}{2} \int_{M} \operatorname{div}\left(\rho \nabla|H|^{2}\right) \eta^{2} \\
& =-\int_{M} \eta \rho \nabla|H|^{2} \cdot \nabla \eta \\
& =2 \int_{M} \eta \rho\left\langle\nabla_{i} H, H\right\rangle \cdot \nabla_{i} \eta \\
& \leq \int_{M}|\nabla H|^{2} \eta^{2} \rho+\int_{M}|H|^{2}|\nabla \eta|^{2} \rho
\end{aligned}
$$

We then have

$$
\int_{M}\left(\frac{1}{2}|H|^{2}+|P|^{2}\right) \eta^{2} \rho \leq \int_{M}|H|^{2}|\nabla \eta|^{2} \rho .
$$


Let $\eta=\phi\left(\frac{|F|}{r}\right)$ for any $r>0$, where $\phi$ is a nonnegative function on $[0,+\infty)$ satisfying

$$
\phi(x)= \begin{cases}1 & \text { if } x \in[0,1) \\ 0 & \text { if } x \in[2,+\infty),\end{cases}
$$

and $\left|\phi^{\prime}\right| \leq C$ for some absolute constant. Since $\nabla z=2 F^{T}$,

$$
\nabla \eta=\frac{1}{r} \phi^{\prime} \nabla \sqrt{z}=\frac{1}{r} \phi^{\prime} \frac{F^{T}}{\sqrt{z}} .
$$

By (1.2) we have

$$
|\nabla \eta|^{2} \leq \frac{C^{2}}{r^{2}} \frac{\left|F^{T}\right|^{2}}{z}=\frac{1}{r^{2} z} C^{2}\left(z+4|H|^{2}\right) .
$$

It follows that (5.2) becomes

$$
\int_{D_{r}}\left(\frac{1}{2}|H|^{2}+|P|^{2}\right) \rho \leq \frac{C^{2}}{r^{2}} \int_{D_{2 r} \backslash D_{r}}|H|^{2}\left(1+\frac{4|H|^{2}}{z}\right) \rho .
$$

By Theorem 4.1 then under the condition on $|H|$, we obtain that the right hand side of (5.3) approaches to zero as $r \rightarrow+\infty$. This implies that $H \equiv 0$.

According to Theorem 3.3 in [16] we see that $M$ is complete with respect to the induced metric from $\mathbb{R}_{m}^{m+n}$. In a geodesic ball $B_{a}(x)$ of radius $a$ and centered at $x \in M$ we can make gradient estimates of $|B|^{2}$ in terms of the mean curvature. From (2.9) in [16] we have

$$
|B|^{2} \leq k \frac{2 m(n-4) a^{2}}{\left(a^{2}-r^{2}\right)^{2}} .
$$

Since $M$ is complete we can fix $x$ and let $a$ go to infinity. Hence, $|B|^{2}=0$ at any $x \in M$ and $M$ is an $n$-plane.

Theorem 5.2. Let $M$ be a complete space-like self-shrinker of dimension $n$ in $R_{m}^{n+m}$. If there is a constant $\alpha<\frac{1}{2}$, such that $\ln w \leq e^{\alpha d^{2}(p, x)}$ for certain $p \in M$, where $d(p, \cdot)$ is the distance function from $p$, then $M$ is affine $n-$ plane.

Proof. (3.15) tells us

$$
\mathcal{L}(\ln w) \geq \frac{|B|^{2}}{w^{2}} \geq 0 .
$$

As an application to (4.4) (Theorem 3.1 in [18]) the Corollary 4.2 in [18] tells us that $\ln w$ is constant. This forces $|B|^{2} \equiv 0$.

\section{REFERENCES}

1. E. Calabi: Examples of Bernstein problems for some nonlinear equations. Proc. Symp. Global Analysis U. C. Berkeley. (1968).

2. A. Chau, J. Chen, and Y. Yuan, Rigidity of Entire self-shrinking solutions to curvature flows, J. reine angew. Math. 664 (2012), 229-239.

3. Qun Chen and Hongbing Qiu: Rigidity theorems for self-shrinker in Euclidean space and Pseudo-Eclideanspace. Preprint. 
4. S. Y. Cheng and S. T. Yau: Maximal spacelike hypersurfaces in the Lorentz- Minkowski spaces. Ann. Math. 104 (1976), 407-419.

5. Xu Cheng and Detang Zhou: Volume estimates about shrinkers, arXiv:1106.4950.

6. Tobias H. Colding and William P. Minicozzi II, Generic Mean Curvature Flow I; Generic Singularities, Ann. Math. 175 (2012), 755-833.

7. Qi Ding and Zhizhang Wang: On the self-shrinking system in arbitrary codimensional spaces. arXiv:1012.0429v2 [math.DG].

8. Qi Ding and Y. L. Xin, Volume growth, eigenvalue and compactness for self-shrinkers, arXiv:1101.1411 [math.DG] (to appear in Asia J. Math.)

9. Qi Ding and Y. L. Xin: The rigidity theorems for Lagrangian self-shrinkers. arXiv:1112.2453 [math.DG] (to appear in J. reine angew. Math.)

10. K. Ecker: On mean curvature flow of spacelike hypersurfaces in asymptotically flat spacetime. J. Austral. Math. Soc. Ser A 55 (1993) no. 1, 41-59.

11. K. Ecker: Interior estimates and longtime solutions for mean curvature flow of noncompact spacelike hypersurfaces in Minkowski space. J. Differential Geom. 46 (1997) no.3, 481-498.

12. K. Ecker: Mean curvature flow of of spacelike hypersurfaces near null initial data. Comm. Anal. Geom. 11no. 2(2003), 181-205.

13. K. Ecker and G. Huisken: Parabolic methods for the construction of spacelike slices of prescribed mean curvature in cosmological spacetimes. Commun. Math. Phys. 135(1991), 595-613.

14. Rongli Huang and Zhizhang Wang, On the entire self-shrinking solutions to Lagrangian mean curvature flow, Calc. Var. Partial Differential Equations 41 (2011), 321-339.

15. Hoeskuldur P. Halldorsson: Self-similar sulutions to the mean curvature flow in the Minkowski plane $\mathbb{R}^{1,1}$. arXiv:1212.0276v1[math.DG].

16. J. Jost and Y. L. Xin: Some aspects ofthe global geometry of entire space-like submanifolds. Result Math. 40 (2001), 233-245.

17. Yung-Chow Wong: Euclidean n-planes in pseudo-Euclidean spaces and differential geometry of Cartan domain. Bull. A. M. S. 75 (1969), 409-414.

18. Guofang Wei and Will Wylie: Comparison Geometry for Bakry-Emery Ricci Tensor, J. Differential Geometry 83(2)(2009), 377-405.

19. Y. L. Xin: Mean curvature flow with bounded Gauss image. Results Math. 59(2011), 415-436.

20. Y. L. Xin: On the Gauss image of a spacelike hypersurfaces with constant mean curvature in Minkowski space. Comment. Math. Helv.66(1991), 590-598.

21. Yuanlong Xin: Minimal submanifolds and related topics. World Scientific Publ. (2003).

22. Y. L. Xin and R. G. Ye: Bernstein-type theorems for space-like surfaces with parallel mean curvature. J. rein angew. math. 489(1997), 189-198.

School of Mathematics and Information Sciences, Henan University, Kaifeng 475004, CHINA.

E-mail address: liuhuaqiao@henu.edu.cn

Institute of Mathematics, Fudan University, Shanghai 200433, China.

E-mail address: ylxin@fudan.edu.cn 